http://jmscr.igmpublication.org/home/ ISSN (e)-2347-176x ISSN (p) 2455-0450 crossref DOI: https://dx.doi.org/10.18535/jmscr/v8i8.48

\title{
To study bacteriological and antibiotic susceptibility profile of burn wound isolates in a tertiary care centre in JAMMU
}

\author{
Authors \\ Dr Perika ${ }^{1}$, Dr Shashi Sudhan Sharma ${ }^{2}$, Dr Priyanka Sharma ${ }^{3}$ \\ ${ }^{1}$ Post Graduate, Department of Microbiology, GMC Jammu \\ ${ }^{2 *}$ Professor, PI and Nodal Officer, VRDL, Department of Microbiology, GMC Jammu \\ ${ }^{3}$ Senior Resident, Department of Microbiology, GMC Jammu \\ *Corresponding Author \\ Dr Shashi S Sharma
}

Professor, PI and Nodal Officer, Dept. of Microbiology, GMC Jammu, India

\begin{abstract}
Burn wounds are one of the most common and devastating form of trauma. Infections are an important cause of morbidity and mortality in burn patients. This was a retrospective study conducted in Microbiology department of GMC JAMMU over a span of 6 months [JAN 2018- JUNE 2018]. Samples transported to microbiology laboratory were immediately processed and antibiotic sensitivity was performed according to standard laboratory procedures (CLSI guidelines). Total of 101 samples were taken from 101 patients admitted in burn ward. Records of these swabs taken from burn wound patients were analysed. The most frequent isolates were Pseudomonas aeruginosa, Klebsiella pneumoniae, Staphylococcus aureus, Proteus. Antimicrobial sensitivity showed Imipenem was most effective drug among Gram negatives and Linezolid was most effective against Gram positive isolates. It is very crucial for every burn unit to determine specific pattern of burn wound colonization and antimicrobial susceptibility pattern. This will enable early treatment of imminent septic episodes with proper empirical systemic antibiotics thus improving overall infection related morbidity and mortality.
\end{abstract}

\section{Introduction}

Infections are an important complication and a major cause of mortality in burn wound patients. A burn is often defined as an injury to the skin or other organic tissue primarily caused by heat or due to radiation, radioactivity, electricity, friction or contact with chemicals. Skin injuries due to ultraviolet radiation, radioactivity, electricity or chemicals, as well as respiratory damage resulting from smoke inhalation, are also considered to be burns $^{(1)}$.
According to the estimates given by World Health Organization (WHO) burn injury results in 265,000 deaths annually, with nearly half of these occurring in the WHO South-East Asia Region ${ }^{(2)}$. Burn wounds are more prone to infection due to destruction of skin barriers and concomitant suppression of immune responses. Also, burn site provides a favourable niche for microbial colonization and proliferation. Now infections are becoming a leading cause of mortality and currently $75 \%$ of all deaths are related to sepsis 
from burn wound infection or other complications due to infections ${ }^{(3)}$.

Various factors favour the growth of microorganisms within the burn site like loss of normal skin barrier at the site, presence of large necrotic tissue and protein -rich wound exudate at the burn surface ${ }^{(4,5)}$. Other risk factors are the size of the burn wound (percentage of total body surface area (TBSA) burnt and the duration of hospitalization $^{(6)}$, extent and depth of injury, colonizing microbe and invasive potential. Skin surface that is intact is vital for the preservation of body fluid homeostasis, thermoregulation, and protection of the host against infection.

Composition of bacteria infecting burn wounds changes depending on duration of burns. Initially, the bacterial flora usually consists of surrounding microbes from the skin, hair follicles, sebaceous glands, and the environment and are usually grampositive Staphylococci or Streptococci. After around day six, these gram-positive organisms are often replaced with gram-negative organisms such as Pseudomonas aeruginosa and Escherichia coli (7)

Antibiotics are commonly used as systemic propylaxis for the management of burn patients ${ }^{(8)}$. The increasing use of antibiotics has led to the emergence of drug resistant bacteria with intrinsic resistance towards antibiotics, ability to survive longer in the hospital environment, hand to hand transmission and these drug resistant bacteria have potential for causing nosocomial outbreaks.

Hence, the present study was planned for a continuous surveillance of predominant flora of the burn wound infections and an estimate of their antibiotic susceptibility pattern to facilitate treatment ahead of microbiology results and aid with preventing further multidrug resistant organisms. This can further help in improving patient care by formulating an updated antibiotic guideline for the management of patients in burn unit.

\section{Materials and Methods}

- Study Design: Retrospective observational study spanning 6 months from JAN 2018JUNE 2018. The records of burn wound samples from patients receiving treatment at Government Medical College \& Hospital, JAMMU were analysed.

- Study Area: Bacteriology Section, Department of Microbiology, GMC JAMMU

- Exclusion/Inclusion Criteria: All patients admitted to burn care unit were included in study. Patients with burns treated on an outpatient basis were excluded.

\section{Sample Collection}

Sample collection was conducted by medical officers in outpatient clinics and in wards using commercially available sterile cotton swabs and following existing departmental guidelines. Two swabs per patient were collected after carefully cleaning wound with sterile water to prevent surface contamination. Samples were transported to microbiology department within one hour of collection to prevent drying of swabs.

\section{Sample Processing}

One swab was used for performing direct staining by Methylene blue and Grams Staining methods as per standard protocols.

Second Swab was immediately inoculated on Blood agar, MacConkey agar, incubated at $37^{\circ} \mathrm{C}$ aerobically for 24-48 hours. Bacterial colonies were identified by colony morphology, Gram's staining and conventional biochemical tests as per standardized protocols of our laboratory.

Different panels of Antimicrobial agents for Gram -positive and Gram-negative bacteria were used as per Clinical Laboratory Standards Institute (CLSI) guidelines. Antibiotic sensitivity was performed by using Kirby- Bauer disc diffusion method. 


\section{Results}

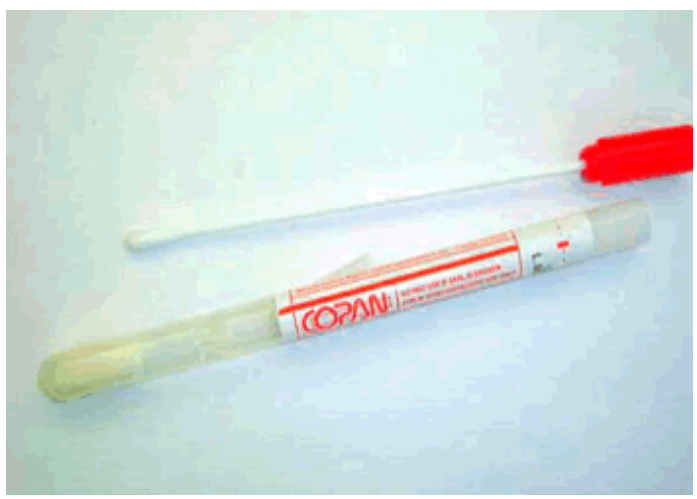

Overall 101 surface swabs taken from 101 patients over a span of 6 months.

A total of 115 organisms were isolated. Pseudomonas aeruginosa was most frequent isolate followed by Klebsiella pneumoniae, Staphylococcus aureus, Acinetobacter, Proteus mirabilis, Enterobacter. [Figure 1]

Figure 1: Wound swab for collection of specimen

Table 1: Results of Surface Swabs

\begin{tabular}{lc}
\hline RESULTS & No. OF SURFACE SWABS \\
\hline Single isolates & $95[82.6 \%]$ \\
Multiple isolates & $12[10.4 \%]$ \\
Sterile & $8[6.95 \%]$ \\
Total & 115 \\
\hline
\end{tabular}

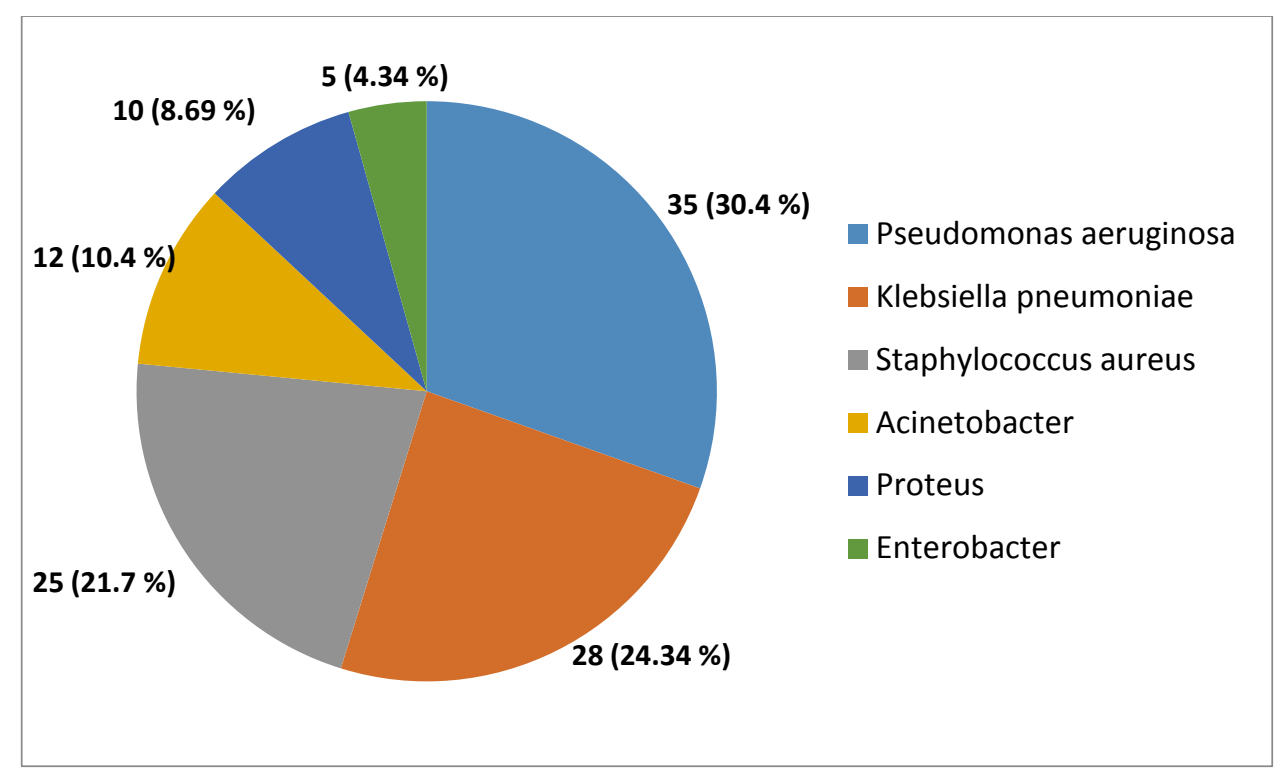

Figure 1: Frequency of microorganisms isolated

Table-2 Antibiotic Susceptibility Pattern of Gram Positive Isolate

\begin{tabular}{|l|c|}
\hline & Staphylococcus aureus ( N=25) \\
\hline Penicillin G & $6(24 \%)$ \\
\hline Cefoxitin & $17(68 \%)$ \\
\hline Gentamycin & $11(44 \%)$ \\
\hline Ciprofloxacin & $12(48 \%)$ \\
\hline Cotrimoxazole & $18(72 \%)$ \\
\hline Vancomycin & $25(100 \%)$ \\
\hline Clindamycin & $22(88 \%)$ \\
\hline Linezolid & $25(100 \%)$ \\
\hline Doxycycline & $21(84 \%)$ \\
\hline Chloramphenicol & $18(72 \%)$ \\
\hline
\end{tabular}


Table 3 Antibiotic Susceptibility of Gram Negative Isolates

\begin{tabular}{|l|c|c|c|c|c|}
\hline & $\begin{array}{c}\text { P. aeruginosa } \\
(\mathrm{N}=35)\end{array}$ & $\begin{array}{c}\text { K.pneumoniae } \\
(\mathrm{N}=28)\end{array}$ & $\begin{array}{c}\text { Acinetobacter } \\
(\mathrm{N}=12)\end{array}$ & $\begin{array}{c}\text { Proteus } \\
(\mathrm{N}=10)\end{array}$ & $\begin{array}{c}\text { Enterobacter } \\
(\mathrm{N}=5)\end{array}$ \\
\hline PIT & $28(80 \%)$ & $15(53 \%)$ & $1(8 \%)$ & $5(50 \%)$ & 0 \\
\hline AmP & $\mathrm{NT}$ & 0 & $\mathrm{NT}$ & $\mathrm{NT}$ & $\mathrm{NT}$ \\
\hline CAZ & $15(43 \%)$ & $11(39 \%)$ & $1(8 \%)$ & $2(20 \%)$ & $1(20 \%)$ \\
\hline CPM & $11(31 \%)$ & $2(7 \%)$ & $3(25 \%)$ & $4(40 \%)$ & $2(40 \%)$ \\
\hline CTR & NT & $4(14 \%)$ & $4(33 \%)$ & $2(20 \%)$ & $1(20 \%)$ \\
\hline AT & $20(57 \%)$ & $22(76 \%)$ & $10(83 \%)$ & $7(70 \%)$ & $4(80 \%)$ \\
\hline IPM & $30(86 \%)$ & $25(89 \%)$ & $11(91 \%)$ & $8(80 \%)$ & $5(100 \%)$ \\
\hline AK & $26(74 \%)$ & $5(18 \%)$ & $9(75 \%)$ & $9(90 \%)$ & $2(40 \%)$ \\
\hline GEN & $22(63 \%)$ & $13(46 \%)$ & $5(42 \%)$ & $9(90 \%)$ & $3(60 \%)$ \\
\hline CIP & $20(57 \%)$ & $4(14 \%)$ & $11(91 \%)$ & $8(80 \%)$ & $4(80 \%)$ \\
\hline COL & $34(97 \%)$ & $22(76 \%)$ & $11(91 \%)$ & $3(30 \%)$ & $4(80 \%)$ \\
\hline POLX & $32(91 \%)$ & $24(86 \%)$ & $9(75 \%)$ & $1(10 \%)$ & $2(40 \%)$ \\
\hline
\end{tabular}

\section{Discussion}

Burn wound infections have been an important cause of nosocomial infections, providing a favourable niche for a multitude of microorganisms to grow. These also pose a major challenge for the clinicians. Infections are the cause of death in $75 \%$ of the burn patients ${ }^{(9)}$. Also, prolonged use of antibiotics has paved the way for the emergence of drug resistant organisms, further posing a challenge for the clinicians in the management of infections. Hence, the present study was planned for the surveillance of bacterial flora and antibiotic susceptibility profile of burn patients so that it would help clinicians in framing appropriate policies for the accurate diagnosis and management of patients in burn unit.

In the present study, a total of 115 organisms were isolated from 101 samples.

In majority of cases $(95,82.6 \%)$, single isolate was seen, while12(10.4\%) cases showed mixed infection. $8(6.95 \%)$ cases were sterile. This was consistent with study by Priyadarshini et al, $\mathbf{2 0 1 8}^{(1)}$ where in majority (96\%) samples tested were culture positive and $4 \%$ cases were bacteriologically sterile.

Our study showed that most frequent isolate was Pseudomonas aeruginosa (30.4\%), followed by Klebsiella pneumonia (24.34\%), Staphylococcus aureus (21.7 \%), Acinetobacter (10.4\%). Infection was predominantly caused by Gram negative organisms. Only one Gram positive organism was found.
Similar observation was seen in the study by Gupta et al, $2019^{(10)}$ where the most commonly isolated organisms were Pseudomonas species (43\%), Klebsiella pneumoniae and Acinetobacter baumannii were second and third predominant bacterial pathogen with a prevalence of $28 \%$ and $14.83 \%$ respectively. Similar finding with Pseudomonas. aeruginosa as a predominant isolate followed by $\mathrm{K}$. pneumoniae in a tertiary care hospital in India were also reported in studies by Dash et al, 2013 ${ }^{(11)}$ and Singh et al, 2003 ${ }^{(12)}$. The high frequency of these pathogens in burn wounds is attributed to their ability to flourish well in a moist environment and persistence in hospital environment.

Our study showed that among Gram positives, Staphylococcus aureus was the most common isolate. This is consistent with studies by Ozumba et al ,2000 ${ }^{(13)}$ and Guggenheim et al , 2009 ${ }^{(14)}$. The variation in the isolation of different organisms among burn wounds is due to the difference in treatment practices in the different geographical locations.

Our study also showed that among Gram positive organisms Linezolid and Vancomycin were the most effective drugs (100\% sensitivity). Among Gram negative infections Imipenem, Colistin, Polymyxin were the most effective drugs. (80$100 \%$ sensitivity). Similar results were seen in the study by Pujji et al, $2019{ }^{(15)}$ wherein sensitivity of Staphylococcus to Vancomycin was $85.7 \%$ and Colistin was highly sensitive among Gram negative infections. Study by 
Priyadarshini et al, $2018{ }^{(1)}$ also showed $100 \%$ sensitivity of Vancomycin and Linezolid for Gram positive while Imipenem was highly effective against Gram negatives.

The antibiogram studies have shown wide degree of resistance to commonly used group of antibiotics like Penicillin group, Cephalosporin group as these antibiotics are being empirically used for prolonged duration. This was consistent with study by Priyadarshini et al, $2018^{(1)}$.

\section{Bibliography}

1. Megha Priyadarshini, Manoj Kumar, Ashok Kumar Sharma, Amber Prashad, Kumari Seema. Bacteriological Profile and Antibiogram of Burn Wound Infections from Burn Patients at RIMS, Ranchi. Int J Med Res Prof. 2018 Nov; 4(6):203-06.

2. World Health Organization. 2017. Burns (online). Available from http://www.who.int/mediacentre/factsheets/fs 365/en/; accessed 2Jun. 2017.

3. Peng DZ1, Liu XL, Liu ZY, Shu WT, Zhou $\mathrm{X}$, Liu $\mathrm{J}$, et al. Analysis of distribution characteristics and drug resistence of 2748 strains of pathogens isolated from burn patients.

4. V G Bhat \& S D Vasaikar (2010) Bacteriological profile and antibiogram of aerobic burn wound isolates in Mthatha, Eastern Cape, South Africa, Southern African Journal of Epidemiology and Infection, 25:4, 16-19.

5. Erol S, Altoparlak U, Akcay MN, Celebi F, Parlak M. Changes of microbial flora and wound colonization in burned patients. Burns 2004; 30: 357-61.

6. Datta S, Ghosh T, Sarkar D, Tudu NK, Chatterjee TK, Jana A. Bacteriological Profile of Burn Wounds and Their Antibiotic Susceptibility Pattern in a Tertiary Care Hospital. Int J Sci Stud 2016;4(5):141-45.
7. Chiller K, Selkin BA, Murakawa GJ. Skin microflora and bacterial infections of the skin. J Investig Dermatol Symp Proc 2001;6:1704.

8. T. Avni, A. Levcovich, D.D. Ad-El, L. Leibovici, M. Paul, Prophylactic antibiotics for burns patients: systematic review and meta-analysis, Bmj 340 (2010 Jan 1)c241.

9. S. Srinivasan, A.M. Vartak, A. Patil, J. Saldanha, Bacteriology of the burn wound at the BaiJerbai Wadia hospital for children, Mumbai, India-a 13-year study, PartI Bacteriological profile, Indian J. Plast. Surg. 42 (2) (2009 Jul) 213, official publication of the Association of Plastic Surgeons of India.

10. Gupta M, Naik AK, Singh SK. Bacteriological profile and antimicrobial resistance patterns of burn wound infections in a tertiary care hospital. Heliyon 5 2019; e02956 .

11. M. Dash, P. Mishra, S. Routray, Bacteriological profile and antibiogram of aerobic burn wound isolates in a tertiary care hospital, Odisha, India, Int. J. Med. Med. Sci. 3 (2013) 460-463.

12. N.P. Singh, R. Goyal, V. Manchanda, S. Das, I. Kaur, V. Talwar, Changing trends in bacteriology of burns in the burns unit, Delhi, India, Mol. Biol. Rep. 29 (2003) 129-133.

13. U.C. Ozumba, B.C. Jiburum, Bacteriology of burn wounds in Enugu, Nigeria, Burns 26 (2000) 178-180.

14. M. Guggenheim, R. Zbinden, A.E. Handschin, A. Gohritz, M.A. Altintas, P. Giovanoli, Changes in bacterial isolates from burn wounds and their antibiograms: a 20year study (1986-2005), Burns 35 (2009) 553-560.

15. Pujji OJ, Nakarmi KK, Shrestha B, Rai SM, Jeffery SL. The Bacteriological Profile of Burn Wound Infections at a Tertiary Burns Center in Nepal. J Burn Care Res 2019; 40(6):838-845. 